\title{
A forced-choice test improves clinical contrast sensitivity testing
}

\author{
VAEGAN $^{1}$ AND B. L. HALLIDAY ${ }^{2}$ \\ From the 'Electrodiagnostic Clinic, Moorfields Eye Hospital, London, and the Institute of Ophthalmology, \\ Judd Street, London; and ${ }^{2}$ Oxford Eye Hospital, Walton Street, Oxford
}

SUMMARY Decreased contrast sensitivity has been demonstrated in early glaucoma, but the deficit is not regularly observed. We designed a prototype for a forced-choice printed test and evaluated it with several other measures of contrast sensitivity. The results also bear on the pattern of loss and the variables which effect performance. Mildly glaucomatous patients show a $6 \mathrm{db}(50 \%)$ loss of contrast sensitivity at all spatial frequencies tested compared with age matched controls. Moving gratings give the same information as stationary ones, and practice effects are negligible. Contrast sensitivity at or below 2 cycles/degree is poorly correlated with visual acuity and does not change with age in the forced-choice test. Subjective judgment made the apparent contrast threshold higher, age dependent, and more variable, particularly at higher spatial frequencies. The pattern of variability can explain some reports of insignificant effects and why low spatial frequency contrast sensitivity detects glaucoma better than visual acuity. Methods correlated so poorly, despite high reliabilities, that uncontrolled biases must be suspected in subjective measures. Our new forcedchoice format was superior to all other tests on at least one formal criterion and always at least equal to them. Improvements in contrast sensitivity screening tests are thus indicated.

There are several recent reports that printed tests of low spatial frequency contrast sensitivity are a sensitive, quick, and clinically convenient indicator of early glaucoma ${ }^{1-6}$ and other eye diseases. ${ }^{278}$ Some studies have obtained significant results only over a limited range of spatial frequencies, ${ }^{2}$ test conditions, ${ }^{4-11}$ or subject ages. ${ }^{812-14}$ Arden $^{2}$ believed that low spatial frequency losses were the most direct index of peripheral retinal damage. In his test greater deficits occur at higher spatial frequencies $(3 \cdot 2$ and 6.4 cycles/deg), where similar losses can be caused by aging and other optical factors. ${ }^{1-15}$ If loss of spatial frequency contrast sensitivity in disease was mainly a consequence of these factors, there would be no clinical advantages in measuring it. The known biases occur in studies which use the rapid but subjective method of adjustment. Forced-choice measures, which are essential to minimise methodological biases, ${ }^{16-19}$ have never been employed.

We devised a simple, rapid, printed, contrast sensitivity test with a psychophysically precise 4-

Correspondence to Dr Vaegan, Electrodiagnostic Clinic, Sydney Eye Hospital, Sir John Young Crescent, Woolloomooloo, Australia 2011. alternative forced choice (4AFC) format. We compared it to an oscilloscope display of gratings, either stationary or phase reversing, and the original printed test ${ }^{12}$ with groups of various ages, including glaucoma patients and age matched elderly normal persons. Our chief purpose was to compare the tests. There are methods specifically designed for this purpose. Normally the same population is tested at least twice with each of the tests to be compared. There will normally be a group which the test aims to discriminate. They should be tested and their scores compared with those of normal control groups. It is then possible to say which is the better test in terms of a number of comparisons between the several retests or groups. These are measures of:

(1) reliability-ordering should change as little as possible on retest (i.e., the correlation of test and retest should approach 1.0$)$; (2) stability-absolute scores should change as little as possible on retest (i.e., the difference between the mean scores of test and retest should approach $0 \cdot 0$ ); (3) generality-the mean and standard deviation should not change with social variables, for example, age, socioeconomic 
group or level of co-operation; (4) efficiency-the separation of the target group (in this case individuals needing clinical attention) from the rest of the population should be as great as possible; (5) utility-the test should be fast and easy to use without sacrificing efficiency.

Our new test always equalled the others and surpassed each of them on at least one of the 5 criteria. By comparing the 4 methods we got additional insight as to why and when contrast sensitivity testing can be of value. A brief report of this research has been published previously. ${ }^{16}$

\section{Methods}

Visual acuities were always tested with printed test charts illuminated to a constant level by bright overhead fluorescent lights. Far visual acuity was determined with a Snellen chart at $6 \mathrm{~m}$ and near visual acuity with a Sheridan-Gardiner single letter series at $30 \mathrm{~cm}$. Subjects wore their appropriate optical correction for each distance. Visual acuity was converted to a ratio which included the proportion of errors on the last visible line, so that small variations could influence the analysis.

We had 3 methods of displaying sinusoidal gratings and a different testing method was required for each one.

(1) Arden's printed test. This test consists of a series of 5 plates with printed reproductions of swept contrast gratings. ${ }^{12}$ (Contrast here signifies the dimensionless ratio $\left(\mathrm{L}_{\max }-\mathrm{L}_{\min }\right) /\left(\mathrm{L}_{\max }+\mathrm{L}_{\min }\right)$ where $\mathrm{L}_{\max }$ and $\mathrm{L}_{\min }$ are the luminances of the brightest and darkest points in the stimulus respectively.) The page edge is marked with a scale, each unit of which is equal to a contrast change of $0.08 \log$ units $(1 \cdot 6 \mathrm{db})$. The scale is placed arbitrarily and runs from 0 , which is below normal threshold, to 20 , which can be easily seen by most visually normal people. Successive plates are separated in spatial frequency by octave steps (doubling of frequency), starting at $0 \cdot 2$ cycles $/ \mathrm{cm}$ and progressing to $6.4 \mathrm{cycles} / \mathrm{cm}$. The 2 highest spatial frequencies are printed on each half of the final plate. The tester first familiarises the subject with the stimulus and then covers it with a grey card of approximately the same reflectance. The card is pulled up the sheet exposing the lowest contrast first, at the rate of about 1 unit/s, until the subject reports he can see the stripes. The number at the margin is recorded. The test takes about $5 \mathrm{~min}$ to administer.

We used the exact procedure specified with the test instructions with 3 exceptions. Firstly, lighting was by the overhead fluorescent lights of the clinic supplemented by that from a nearby window and not by a desk lamp directly above, since we found that there was less diffuse reflection from the page (glare) and that we obtained the same result without the additional illumination. Secondly, the test distance was $28.5 \mathrm{~cm}$ and not $57 \mathrm{~cm}$, since this was closer to the patients' reading distance. The spatial frequencies studied thus range from $0 \cdot 1$ to $3 \cdot 2$ cycles/deg. Finally, observers who failed to detect any grating were given a nominal score of 21 for the purpose of analysis, rather than 25 , since a group of scores so distinct from the rest would have skewed the distribution of scores in some groups and thus increased variance excessively.

(2) The 4-alternative forced-choice (4AFC) test. The Arden plates were cut into a series of discs. Each disc differed from the next by one scale unit (see above). Disc diameter was $7 \mathrm{~cm}$ for plates 2 to $5(0 \cdot 2$ to $1.6 \mathrm{cycles} / \mathrm{cm})$, which therefore varied from scale level 3 to 17 and $3 \mathrm{~cm}$ for plates 6 and $7(3.2$ and 6.4 cycles $/ \mathrm{cm}$ ), covering levels 2 to 19 . With these sizes contrast varied little within a plate, and there were enough grating cycles on the disc (except that there were only 2 cycles for the coarsest grating). Each series was mounted on a grey card of similar mean reflectance, high contrasts first. Because the sweep direction defines a top there are 8 orientations differing from vertical in steps of 45 degrees. Each was used once in random order before the set was repeated. The subjects were aware of only 4 possible orientations-horizontal, vertical, and left and right oblique-which they had to identify.

We used precisely the same viewing conditions for the 4AFC and Arden tests. In demonstration and practice trials the tester described the 4 possible answers then asked the subject to tell him which way the stripes ran on the highest contrast disc at a moderate spatial frequency, where most people are most sensitive. Children as young as $\mathbf{3}$ years of age could follow the stripes with their finger and thus demonstrate adequate co-operation. Older people used a combination of naming and pointing. On test trials the various spatial frequencies were always presented in either an arbitrary or prearranged random order depending on the particular study. Observers worked down through the contrast scale until they made an error. A second guess was always elicited, because it was unlikely to be correct by chance alone $(p=1 / 3)$. The threshold was taken as the disc preceding that on which the observer made 2 errors. Testing time was about twice as long as the Arden test (i.e., $10 \mathrm{~min}$ ) but could be much longer with some uncooperative children.

The 4AFC test design improvement relies on its great formal similarity to clinically established visual acuity tests like the Sheridan-Gardiner, Snellen, Illiterate E, and Sjögren hand. There are several distinct responses and the tester always knows, from an answer sheet if need be, whether answers are right or wrong. Previously described clinical tests of 
contrast sensitivity have never incorporated objective scoring, even when utilising oscilloscope displays under computer control. Certainly no previous printed test ${ }^{1220}$ has these features. (Atkinson et al. ${ }^{21}$ have recently reported one such test. It was being developed at the same time as ours but has a purely research-orientated format and application.)

(3) Oscilloscope with manual contrast control. Gratings were generated directly on the face of a Telequipment D83 oscilloscope by the television technique of Schade ${ }^{22}$ as modified by Robson. ${ }^{15}$ When phase reversing and standing gratings were compared, the pattern was determined by a microprocessor controlled grating generator. ${ }^{23}$ Subjects could set the contrast with a potentiometer placed near their right hand. Equal increments of rotation produced equal increments of log contrast over a range of $2 \log$ units $(40 \mathrm{db})$. Contrast could be read directly in $\mathrm{db}$ from a digital voltmeter, visible only to the tester. Additional fixed attenuation of 10 or $20 \mathrm{db}$ was often used when the more sensitive young subjects were tested. Spatial frequency could be read directly from a meter and was continuously adjustable. The subject sat with his or her chin on a chin rest, which ensured the eyes were positioned $28.5 \mathrm{~cm}$ from the display. The screen subtended $26 \times 20$ degrees at the eye and was approximately 100 candelas $/ \mathbf{m}^{2}$ in luminous emittance. Frame rate was $100 \mathrm{~Hz}$. Contrast of the screen was within the linear range for the spatial frequencies used, which were always below 12 cycles/deg. After the first experiment the distance and spatial frequencies used were the same as those for the printed tests.

The test was described and demonstrated to the subjects, who were then given some practice at controlling contrast. On each trial a new spatial frequency was selected. Subjects were asked to turn the contrast rapidly towards maximum and to turn it down again as soon as they had seen the stripes clearly. They then turned the contrast up smoothly, slowly, and evenly until the stripes first appeared. It was explained that at this point the pattern would be difficult to see and might come and go. (It should be noted that we did not determine a descending contrast threshold. In common with other laboratories (R. Hess, personal communication) we find that descending settings are much more variable. Many subjects cannot decide when the grating has finally disappeared and, even with a logarithmic contrast control, will turn it to the maximum excursion, far below any likely threshold. These errors increase with rate of change.) We did not permit the subject to make any further contrast adjustments (bracketing). The tester watched the meter to ensure that the rate of contrast change was always about $0.5 \mathrm{db} / \mathrm{s}$ and corrected the subjects if they turned the contrast knob too rapidly. Each of the 6 or 7 stimuli used in the experiment was presented once in random order. The set was then repeated. There were 4 sets in experiment 1 and 5 in experiment 2. Each set took about $10 \mathrm{~min}$ to complete and instruction a further 10 .

\section{Subjects}

Subjects were volunteers from the patients and staff of New Addenbrooke's Hospital, Cambridge. Glaucomatous subjects were patients at the Eye Clinic. Most normal observers, including the unrelated, age matched, elderly normal group, were people who accompanied them. Some inpatients who did not have eye disease were also tested. All people accepted for testing wore their normally prescribed optical correction (if any) for near vision. All testing was monocular. The untested eye was patched. The normal persons had no signs or symptoms of eye disease and no family history of glaucoma. All patients had an ocular examination on the day of the test. We did not test those patients whose notes indicated they had severe glaucoma or bilateral field losses which included the macula. No eye drops, other than normal medication, were applied prior to testing. Other criteria for the selection of patients varied and are given before the 2 studies where clinical groups are involved.

\section{Experiment 1}

There were reports that the loss originally found with the Arden test ${ }^{12}$ could not be detected with static oscilloscope displays ${ }^{9}$ or when elderly glaucoma patients were compared to an age matched control group. ${ }^{14}$ We therefore first compared the contrast sensitivity of glaucoma patients and age matched normal controls, using an oscilloscope to display static gratings at a wider range of spatial frequencies than were available with the printed tests.

\section{METHOD}

To minimise the degree of glaucoma and normal age change we only tested the better eye of each subject, for example, that eye with the least field loss or with the best visual acuity when field losses were equal or absent. Contrast sensitivities were measured by the procedure described above at a spatial frequency of 0.25 cycles/deg and in octave steps to 8 cycles/deg. Measures were also made with 2 cycle/deg square wave luminance profile stripes*. We measured 4 sets *Dr J. Robson had predicted that contrast sensitivity losses in glaucoma would not depend on the spatial frequency composition of the stimulus, for example, the difference obtained when testing with sinusoidal gratings should be no larger than that which occurred when square waves, which contain additional high spatial frequency components, were used. This was confirmed. 
Fig. 1 Means and standard deviations for all 3 groups at all 6 spatial frequencies tested with sinusoidal luminance profile gratings and with square wave luminance profile gratings at 2 cycles/deg. Note that the loss in glaucoma is roughly equal at all spatial frequencies and there is an associated deficit in near visual acuity. Although the loss is significant when the 2 groups are compared across spatial

frequencies, separate comparisons at higher spatial frequencies become less significant because variance increases. The acuity difference is significant, as is the drop in contrast sensitivity with age.

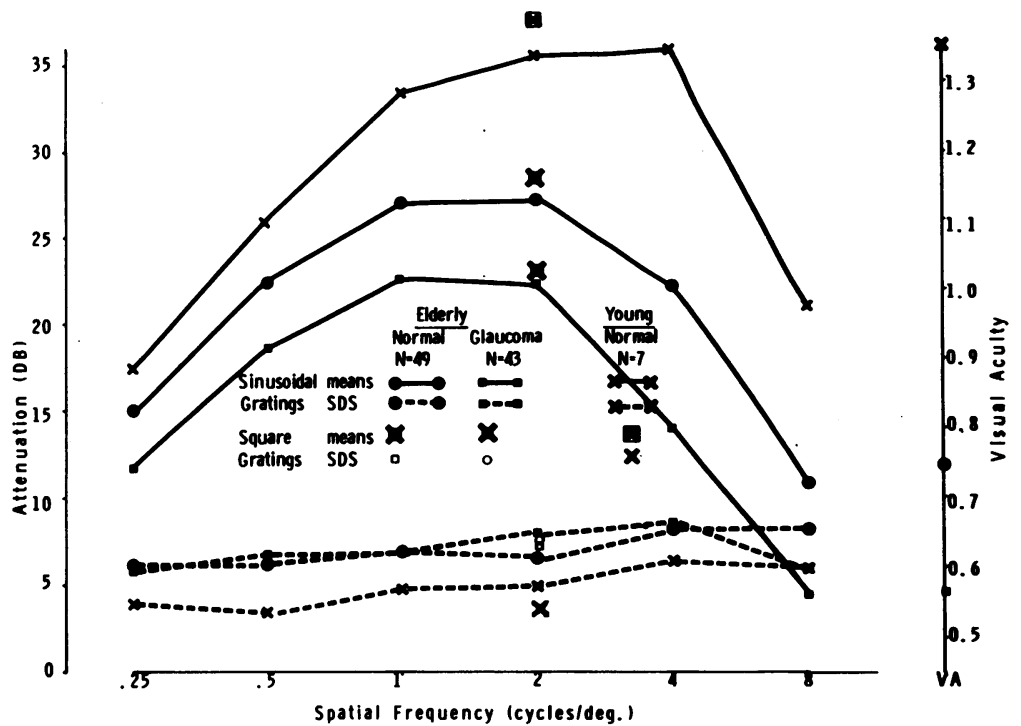

more severe field losses were included because they had in general retained better visual acuity than some of the apparently less severe field losses. Mean maximum recorded intraocular pressure was 29 $\mathrm{mmHg}$ (SD 6.8, range 20-45) and on the testing day the mean was $18.6 \mathrm{mmHg}$ (SD 5.4, range 10-38). Of the 19 cases in which pressure was surgically controlled, mostly by trabeculectomy and in some cases by peripheral irredectomy, only 5 received ocular medication (acetazolamide in 2 cases). In 17 of the other 24 cases some medication was used. No drug was used by $23 ; 13$ took pilocarpine in various doses and therefore had miotic (very small) pupils when tested; 5 took timolol and 2, as noted, acetazolamide. Ocular complications among the patients were normal for this age group: 8 had early cataract, 1 was aphakic, 1 had a mild diabetic retinopathy, 1 had previously suffered from scleritis, and 1 had undergone cryosurgery to prevent retinal detachment such as had already occurred in the fellow eye.

\section{RESULTS}

Practice caused no change at all in the average threshold at any spatial frequency for any age group. Fig. 1 shows, for all groups, average contrast sensitivity and its standard deviation. Average near visual acuity is on the right margin. The maximum sensitivity (36 db or 1.6\%) and extrapolated cut off spatial frequency ( 23 cycles/deg) of the young adults is similar to a comparable, normal, naive group. ${ }^{24}$ (In laboratory experiments sensitivity can be above $40 \mathrm{db}$ attenuation (under $1 \%$ contrast) and cut off spatial frequency above 30 cycles/deg (1 min arc). Scores (nearest point to the macula 18-25 deg), moderate constricted in 8 (12-18 deg), a large step in 9 (4-15 $\mathrm{deg})$, and very constricted in 3 (2-5 deg). Those with 
were lower in our conditions because contrast was turned up on all trials (ascending method of adjustment), bracketing was not permitted, and the observers were unpractised.)

As spatial frequency increased, a large number of elderly people, particularly patients, said they could not see gratings well within the resolution limit expected from their visual acuities. This is why we did not test higher spatial frequencies. Furthermore, even with the 8 cycles/deg stimulus they inspected the target at $100 \%$ contrast for some time before saying they could see the grating. Slow responding is common to all elderly people, and when the contrast can no longer change it further biases the measure.

Analysis of variance of the elderly subjects' scores shows that the glaucomatous group have a significant $6 \mathrm{db}$ sensitivity loss (a factor of 2 ) for all stimuli. There is a slight but significant tendency for differences to increase with spatial frequency and the patients also have a significantly lower visual acuity. The scores of the elderly normal persons and glaucomatous patients overlap substantially. If we set a rather lax false positive rate of about $6-10 \%$, the $0.25 \mathrm{cycle} / \mathrm{deg}$ stimulus picks up $30 \%$ of the glaucomas. The correct assessment increases with spatial frequency to $44 \%$ with $8 \mathrm{cycles} / \mathrm{deg}$, but this is a trivial consequence of the biased behaviour at higher spatial frequencies noted above. If the aggregate over spatial frequencies is used, the discrimination is marginally better ( $42 \%$ correct assessments to $6 \%$ false positives). These figures are of course only approximations and depend critically on the chosen cut-off in what is still a relatively small, mildly affected group in which only the better eye was tested.

When the young and elderly normal subjects were compared (Fig. 1), older people's sensitivity was 3-15 $\mathrm{db}$ lower and standard deviations were larger. The different behaviour at higher spatial frequencies noted above must contribute to this pattern. The standard deviation of scores increased by $50 \%$ with spatial frequency in all groups.

The average correlations between all variables are shown in Table 1. Because each setting was repeated 4 times there were 6 separate pairs from which reliabilities were calculated (on diagonal cells) and 16 pairings of any 2 spatial frequency settings to estimate their correlation (off diagonal cells). The reliability of the measure at any spatial frequency is about $0 \cdot 75$. The increased reliabilities at higher spatial frequencies is simply due to some subjects slowly responding to maximum contrast, as noted above. Not surprisingly, the correlation between sensitivity scores increase as the spatial frequencies at which the measures were made become more similar. In contrast, the correlation between visual acuity and contrast sensitivity is low and equal for all spatial frequencies. It is more highly correlated with age, an observation which will recur later. The correlation of sensitivity with age is $-0 \cdot 1$ at 0.25 cycle/deg and -0.51 at 8 cycles/deg. This shows that contrast sensitivity decreases as age increases and the effect is more marked at higher spatial frequencies.

\section{DISCUSSION}

Our results are in accord with most studies using printed tests but disagree with the findings of Atkin et al. ${ }^{9}$ and Sokol et al. ${ }^{14}$ Glaucoma patients do have a contrast sensitivity deficit of about $6 \mathrm{db}$ in relation to age matched normal persons at all spatial frequencies when tested with a conventional oscilloscope display of static gratings. We also found that visual acuity and contrast sensitivity for square wave gratings were similarly reduced in glaucoma by about $6 \mathrm{db}$. There is evidence that luminance gradients determine low spatial frequency detection. ${ }^{25}$ The central retina would be able to do this best. Our data similarly are against the view that peripheral retinal damage is especially revealed by tests at low spatial frequencies ${ }^{2}$

Table 1 Average correlation between repeated oscilloscope settings to standing sinusoidal gratings at 6 spatial frequencies, plus age and visual acuity. On the diagonal are means of 6 reliability estimates $(4 \times 3 / 2)$ and off it means of 16 correlations (4×4). Reliabilities at high spatial frequencies are artificially high for reasons given in the text. Correlation drops systematically as stimulus differences increase. Visual acuity fails to follow this general trend and correlates better with age.

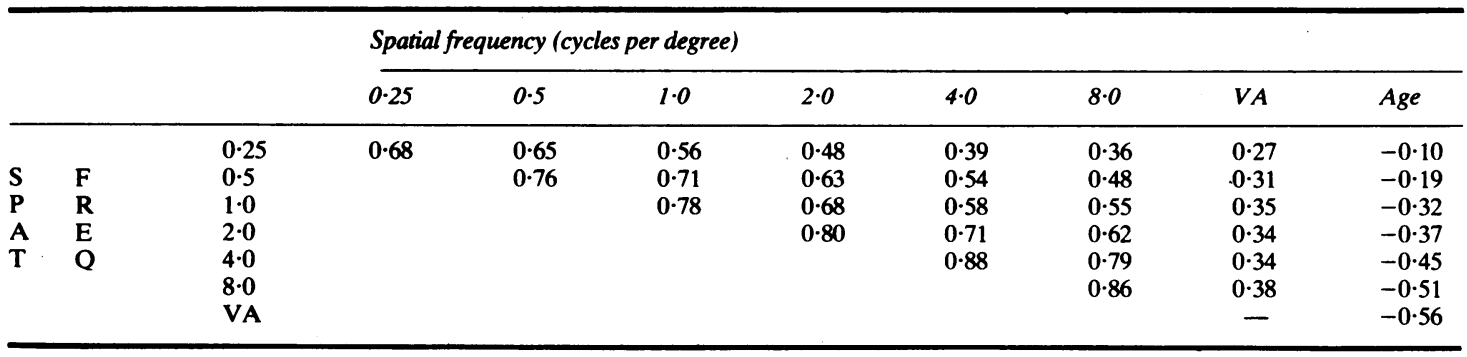

SPAT/FREQ in left-hand column=spatial frequency (cycles per degree). 
but do show why these stimuli are clinically useful.

The significant loss of visual acuity we found associated with glaucoma was so small as to be of no clinical importance in any individual case. Visual acuity and cut off spatial frequency are 2 measures of the resolution limit. Our observation that they are often poorly correlated and may measure largely separate abilities has been made previously. ${ }^{26}$ In disease either may be more severely reduced. ${ }^{26} 27$ When the cause of this discrepancy is known, comparing both measures may be clinically useful. Sensitivities for quite moderate spatial frequencies (above 2 cycles/deg) $)^{11}$ as well as the resolution limit ${ }^{152}$ can be seriously affected by many optical and neural variables which are hard to isolate and control. Gratings of still lower spatial frequencies are clinically useful because they are less sensitive to these factors.

Low spatial frequencies offer advantages for other reasons too. We used what are ordinarily considered quite moderate spatial frequencies. Nevertheless we found that as spatial frequency increased so did variability. These increases were associated with behavioural changes and were greater for older people. For this reason alone disease may cause larger deficits at higher spatial frequencies, but larger samples will be necessary to detect them. This might explain why visual acuity is sometimes said to be unchanged in glaucoma and why some studies have not found significant differences. The significance of loss of contrast sensitivity at any spatial frequency will therefore depend on sample size and the degree to which these biases are present in the test used. Nevertheless we found that these high frequency biases were not very evident when square wave luminance profile bars were used. If this were to be confirmed, future clinical contrast sensitivity tests could be considerably simplified.

Contrast sensitivity, measured by the Arden test, decreases with age..$^{12-1416}$ These oscilloscope settings also decrease with age, as do those of Beasley et al. ${ }^{24}$ We believe that age trends may be greater with subjective methods, for reasons given below.

\section{Experiment 2}

Having confirmed that glaucoma patients do show contrast sensitivity loss, we wanted to establish what method of testing contrast sensitivity would provide the most clinically useful index of reduced function. There are obvious advantages to printed tests, and we wanted to see if the 4AFC format described above justified further development. There are 5 criteria, defined above, by which tests can be formally and experimentally evaluated. ${ }^{29} 30$ Three separate studies were necessary before comparisons could be made using all 5 criteria. In this experiment the test results of a large group of normal people were analysed to see how the several tests compared. In experiment 3 we repeatedly tested normal people with the 2 printed tests to see if the scores of an individual changed. Finally in experiment 4 we looked at the effect of age and glaucoma on the printed tests results to see which was most useful in practice.

\section{METHOD}

Thirty-six normal people of a wide range of ages (mean $35 \cdot 2$ years, SD $21 \cdot 2$, range 5-82) were tested on the 2 printed tests and the oscilloscope display with static and $4 \mathrm{~Hz}$ phase reversing gratings set to the same 6 spatial frequencies using the same $28.5 \mathrm{~cm}$ testing distance. Test procedure and subject selection were as described above. In each test, gratings of the various spatial frequencies were presented in random order. Each of the 4 tests was completed before another, selected at random, began. Both eyes were tested successively, and the order was reversed for each test. With the oscilloscope test 5 threshold values were obtained and averaged. Visual acuity at 'near' (Sheridan-Gardiner Singles) (mean 0.75, SD $0 \cdot 22$, range $1 \cdot 0-0.25$ ) and 'far' (Snellen) (mean 0.92, SD 0.34 , range $2 \cdot 0-0 \cdot 25$ ) was also recorded. For statistical purposes all 72 eyes were treated separately. There were scores on 27 variables for each eye ( 4 methods $\times 6$ spatial frequencies, plus 2 visual acuities and age).

We made the measurements in this way because such data are best suited to a later analysis which can tell us about the relationship between the tests. The average score of the group on each variable is of little interest, because any measure is made only once and any treatment which might change it is avoided. Instead the first step is to calculate all possible correlations between variables. In this experiment there were 351 of them $(27 \times 26 / 2)$. Such a large set of numbers is incomprehensible unless general patterns can be found. Some obvious patterns can be shown by averaging subsets of the correlation matrix.

Factor analysis ${ }^{30}$ is a more precise way of describing these interrelations. It is a mathematical method for formally analysing the correlation matrix, which reduces a large number of variables to a smaller set of hypothetical sources of variation (called factors). Highly correlated variables share more factors in common. Each variable is given a loading on each factor which shows how the variable would correlate with the factor if it could be directly measured. The experimenter can then characterise the factor from what he knows about the variables. If the raw scores are multiplied by the factor loadings each subject can be given a score on each factor so that a large number of test scores can be reduced to a much smaller 


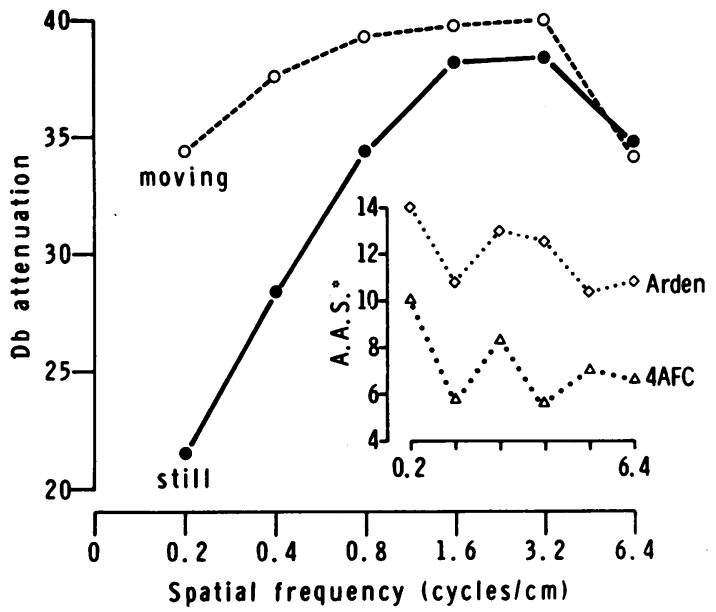

Fig. 2 Average scores on the 4 contrast sensitivity tests for all 74 eyes in experiment 2 . Scores on the printed tests do not converge at high spatial frequencies as the 2 oscilloscope test scores do, which suggests that the systematic difference between them is not associated with movement cues caused by different inspection strategies. Contrary to the $d b$ attenuation scale, higher scores on the arbitrary scale provided with the printed tests imply poorer performance.

number of factor scores, considerably simplifying later analysis.

\section{RESULTS}

The investigation was not directly concerned with the average scores. They are presented in Fig. 2 merely to show that the values are normal. The static grating results are similar to the young adults' scores in the previous experiment and other studies as discussed above. The different curves found with static and phase reversing gratings, especially at low spatial frequencies, may also be expected from previous

Table 2 Average across all tests of the correlation between 2 spatial frequencies in the same test. Each number is the mean of 6 correlations $(4 \times 3 / 2)$. Signs have been inverted for all results involving the Arden or $4 A F C$ test so as to eliminate the negative correlations which would otherwise result, because higher scores on these tests imply worse sensitivity. The correlation generally decreases slowly with increasing difference in spatial frequency.

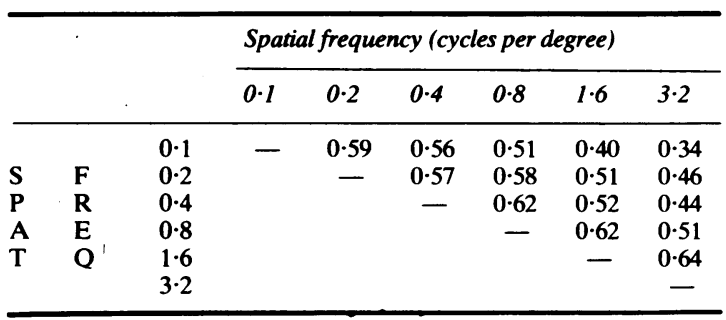

work. ${ }^{31}$ The 2 printed test curves are shown as an inset. Note that here higher scores imply less sensitivity. The means and standard deviations we obtained on the Arden test are very similar to those in previous studies ${ }^{12}$ despite the minor procedural changes.

Table 2 shows the average over all tests of correlations between spatial frequencies. The same pattern was found for each test. As in the previous experiment (Table 1) all correlations are high, and closer spatial frequencies were more highly correlated.

Table 3 gives the average correlation between tests irrespective of spatial frequency. The mean correlation between scores at 2 different spatial frequencies in the same test (on diagonal cells) is much greater than that between all scores from different tests (off diagonal cells). (Because correlations are high within a test the numbers in the cells on the diagonal of Table 3 are a function of each method's reliability but must be smaller than the correct figure. Thus the previous estimate of reliability for a single oscilloscope setting $(0 \cdot 68-0 \cdot 88$, Table 1) is greater than the average over all spatial frequencies here $(0 \cdot 61)$. A comparison is possible

Table 3 Average correlation between all test pairs across all spatial frequency pairs. On the diagonal is the average of the 15 correlations between spatial frequencies within a test $(5 \times 6 / 2)$. Because the correlation decreases slowly with increasing difference in spatial frequency (Tables 1 and 2), these figures are lower bound estimates of reliability, even though the correlation of a spatial frequency with itself is excluded. Thus the visual acuity 'reliability' is only the correlation of measures at near and far. Each number off the diagonal is based on 36 between test correlations. The correlations remained relatively constant where each plate came from a different test and are generally lower than those between widely separated spatial frequencies from the same test. Standard deviations were about $0 \cdot 1$, and there was less systematic variation with difference in spatial frequency than for within-test correlations.

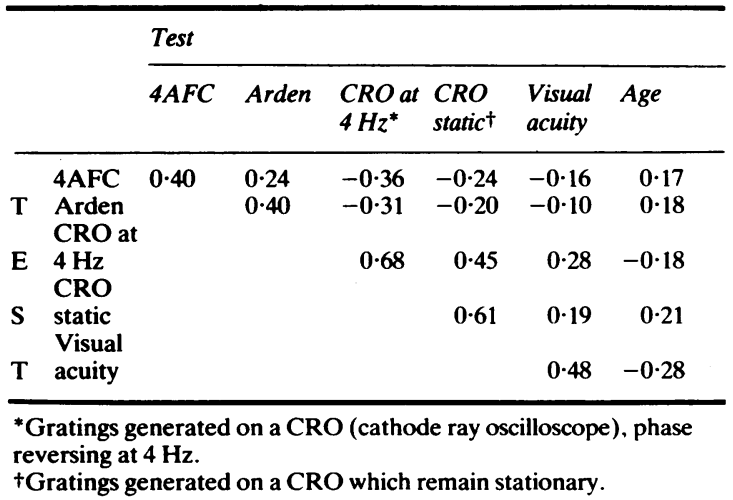


Table 4 Orthogonal, varimax rotated factor structure obtained for 74 eyes tested on 27 variables in experiment 2 . All loadings of 0.3 or more have been printed in bold so that the pattern of loading on each factor can be seen at a glance. Similar solutions for the relationship between the 2 printed tests, visual acuities, and age was found in all subsequent sets of data.

\begin{tabular}{|c|c|c|c|c|c|c|c|c|}
\hline \multicolumn{2}{|c|}{$\begin{array}{l}\text { Factor } \\
\text { Variable (cy/deg) }\end{array}$} & \multirow{2}{*}{$\begin{array}{r}\begin{array}{r}C R O \\
\text { static }\end{array} \\
0.21\end{array}$} & \multirow{2}{*}{$\begin{array}{l}\begin{array}{l}\mathrm{CRO} \\
\text { at } 4 \mathrm{~Hz}\end{array} \\
-0.02\end{array}$} & \multirow{2}{*}{$\begin{array}{r}4 A F C \\
0.67\end{array}$} & \multirow{2}{*}{$\begin{array}{r}\text { Arden } \\
0.30\end{array}$} & \multirow{2}{*}{$\begin{array}{c}\begin{array}{l}\text { Arden } \\
\text { High SF }\end{array} \\
0 \cdot 11\end{array}$} & \multirow{2}{*}{$\begin{array}{c}\begin{array}{l}\text { Near } \\
\text { blur }\end{array} \\
0 \cdot 12\end{array}$} & \multirow{2}{*}{$\begin{array}{c}\begin{array}{l}\text { Visual } \\
\text { acuity }\end{array} \\
-0.28\end{array}$} \\
\hline & $0 \cdot 1$ & & & & & & & \\
\hline 4 & $0 \cdot 2$ & $0 \cdot 13$ & -0.04 & 0.64 & 0.13 & $-0 \cdot 20$ & $0 \cdot 08$ & $-0 \cdot 10$ \\
\hline A & 0.4 & $0 \cdot 12$ & -0.27 & 0.74 & $0 \cdot 19$ & 0.07 & $-0 \cdot 13$ & $-0 \cdot 02$ \\
\hline $\mathrm{F}$ & 0.8 & $-0 \cdot 01$ & -0.32 & 0.73 & $0 \cdot 12$ & $-0 \cdot 12$ & $-0 \cdot 22$ & $0 \cdot 10$ \\
\hline C & 1.6 & -0.02 & $-0 \cdot 37$ & 0.64 & $-0 \cdot 19$ & -0.27 & $-0 \cdot 13$ & $0 \cdot 12$ \\
\hline & $3 \cdot 2$ & $0 \cdot 29$ & $-0 \cdot 21$ & 0.33 & 0.00 & $-0 \cdot 23$ & -0.65 & $-0 \cdot 04$ \\
\hline $\mathbf{A}$ & $0 \cdot 1$ & -0.11 & $-0 \cdot 17$ & $0 \cdot 26$ & 0.77 & -0.02 & $-0 \cdot 06$ & $0 \cdot 04$ \\
\hline $\mathbf{R}$ & $0 \cdot 2$ & $0 \cdot 17$ & $-0 \cdot 09$ & $0 \cdot 28$ & 0.66 & -0.34 & $0 \cdot 07$ & $0 \cdot 11$ \\
\hline D & 0.4 & 0.04 & -0.02 & -0.07 & 0.83 & 0.00 & $-0 \cdot 06$ & $-0 \cdot 17$ \\
\hline $\mathbf{E}$ & $0 \cdot 8$ & $0 \cdot 11$ & $-0 \cdot 13$ & $0 \cdot 20$ & 0.62 & $-0 \cdot 24$ & $-0 \cdot 18$ & $-0 \cdot 24$ \\
\hline $\mathbf{N}$ & $1 \cdot 6$ & 0.30 & $-0 \cdot 16$ & 0.06 & 0.33 & -0.74 & $-0 \cdot 08$ & -0.02 \\
\hline & $3 \cdot 2$ & 0.06 & -0.30 & $0 \cdot 20$ & $0 \cdot 12$ & $-0 \cdot 72$ & $-\mathbf{0 . 3 0}$ & $-0 \cdot 12$ \\
\hline & $0 \cdot 1$ & $-0 \cdot 15$ & 0.79 & -0.26 & -0.22 & $0 \cdot 10$ & -0.08 & $-0 \cdot 04$ \\
\hline C 4 & $0 \cdot 2$ & -0.34 & 0.79 & $-0 \cdot 19$ & -0.08 & 0.02 & 0.02 & $0 \cdot 17$ \\
\hline $\mathbf{R}$ & 0.4 & -0.25 & 0.80 & $-0 \cdot 17$ & -0.05 & 0.08 & $0 \cdot 06$ & $0 \cdot 13$ \\
\hline $\mathrm{O} H$ & 0.8 & -0.31 & 0.72 & -0.30 & $-0 \cdot 12$ & $0 \cdot 27$ & $0 \cdot 17$ & $0 \cdot 03$ \\
\hline at $z$ & $1 \cdot 6$ & -0.40 & 0.64 & -0.15 & $-0 \cdot 14$ & $0 \cdot 34$ & $0 \cdot 22$ & $0 \cdot 22$ \\
\hline & $3 \cdot 2$ & -0.34 & 0.59 & $-0 \cdot 05$ & $-0 \cdot 14$ & $0 \cdot 15$ & 0.48 & $0 \cdot 20$ \\
\hline S & $0 \cdot 1$ & -0.71 & 0.34 & -0.04 & -0.03 & $-0 \cdot 28$ & $0 \cdot 18$ & $-0 \cdot 01$ \\
\hline C $\mathrm{T}$ & $0 \cdot 2$ & -0.79 & 0.35 & $-0 \cdot 06$ & $-0 \cdot 20$ & $-0 \cdot 01$ & $-0 \cdot 07$ & $0 \cdot 00$ \\
\hline R A & 0.4 & -0.81 & 0.33 & $0 \cdot 00$ & $-0 \cdot 11$ & 0.03 & $0 \cdot 05$ & -0.05 \\
\hline O T & 0.8 & -0.78 & $0 \cdot 13$ & $-0 \cdot 12$ & $0 \cdot 02$ & 0.25 & $0 \cdot 20$ & $-0 \cdot 10$ \\
\hline I & $1 \cdot 6$ & -0.78 & 0.05 & $-0 \cdot 16$ & 0.07 & 0.37 & $0 \cdot 13$ & $0 \cdot 19$ \\
\hline C & $3 \cdot 2$ & -0.68 & $0 \cdot 19$ & -0.20 & 0.04 & $0 \cdot 27$ & $0 \cdot 30$ & $0 \cdot 23$ \\
\hline Visual & Far & $0 \cdot 00$ & $0 \cdot 24$ & $-0 \cdot 14$ & $0 \cdot 09$ & 0.07 & $0 \cdot 07$ & 0.84 \\
\hline Acuity & Near & $-0 \cdot 15$ & $0 \cdot 09$ & $0 \cdot 12$ & -0.09 & $0 \cdot 10$ & $0 \cdot 67$ & 0.57 \\
\hline Age & & $0 \cdot 11$ & $0 \cdot 02$ & 0.03 & $0 \cdot 10$ & -0.06 & -0.88 & $0 \cdot 03$ \\
\hline$\%$ Varia & & 16 & 16 & 11 & 10 & 8 & 9 & 6 \\
\hline
\end{tabular}

because all estimates are similarly reduced, but both oscilloscope scores may appear more reliable than either of the printed test scores only because they are an average of 5 settings.) The correlation was always small and did not clearly increase for closer spatial frequencies when they were presented in different formats. Nevertheless all these measures of contrast sensitivity are yet more poorly correlated with visual acuity. It correlates best with age. The same pattern was found in experiment 1 (Table 1). Thus low spatial frequency contrast sensitivity can be regarded as a distinct ability, although there are other nonrandom variables, peculiar to each test, which affect the score.

Similar conclusions can be more directly derived from a factor analysis of the data from the current experiment (Table 4). Factor loadings of 0.3 or greater are printed in bold. We can then clearly see a pattern in which a major factor is associated with each testing method, the oscilloscope tests are very similar, and the 2 printed tests have more in common with either oscilloscope test than with each other. There are 3 minor factors all concerned with visual acuity. One is clearly 'pure' visual acuity, since it loads on the highest spatial frequencies and both visual acuity tests. The next is involved with 'near' vision, high spatial frequencies, and changes with age (apparently presbyopia but called 'near blur' because it is also found in children's data in other studies). The last is peculiar to the Arden test.

To identify features which might affect the result we made a list of all the recognisable differences between the 4 tests (Table 5). No single feature easily explains the obtained pattern of correlations, but if the total number of countable differences is low, the correlation tends to be higher. Therefore any set of methodological difference may be important.

\section{DISCUSSION}

An individuals's performance on contrast sensitivity tests with gratings of 1.6 cycles/deg or lower is so loosely related to both visual acuity and optical 
Table 5 Systematic differences between the 4 contrast sensitivity tests used in experiment 2 which might influence the way subjects respond.

1. Stationary (4AFC, A,S) vs. moving stimuli (M)

2. Accuracy checked (4AFC) vs. subjective psychophysics $(\mathrm{S}, \mathrm{M}, \mathrm{A})$

3. Descending (4AFC) vs. ascending (A,S,M) contrast series

4. Some oblique (4AFC) vs. all vertical (A,S,M) grating orientation

5. High spatial frequencies in small (4AFC) vs. large (A,S,M) areas

6. Low spatial frequencies have few (4AFC) or many (A,S,M) cycles

7. Smooth pursuit (A) vs. free search (4AFC,S,M)eye movements

8. Viewing time limited (A,S,M) vs. free (4AFC)

9. Patterns printed, dull and reflected $(4 \mathrm{AFC}, \mathrm{A})$ vs. electronic, bright and emitted (S,M)

10. Well lit room (4AFC,A) vs. dark room $(S, M)$

11. Examiner directed (4AFC, A) vs. self-controlled (S,M)

$\mathrm{S}=$ static gratings on the oscilloscope. $\mathrm{M}=$ moving gratings on the oscilloscope. $A=$ Arden's test.

factors $^{15}$ that it must indicate a distinct ability. However, there are such substantial differences between the subject rankings obtained in the various tests that we cannot decide which of them is the best measure.

The 2 tests employing the oscilloscope display give results which are closely related statistically, even though the appearances of static and contrast reversing gratings are very different; the difference between the means varies systematically with spatial frequency, and it is often thought that they can differentially stimulate separate visual channels. ${ }^{32}$ On the other hand the 2 tests where the stimuli are cut from the same printed sheets give poorly related scores when only the layout and testing method differed.

\section{Experiment 3}

A clinical test should be as accurate as possible. In terms of the formal criteria defined above there are 2 sorts of accuracy to consider: reliability and stability. We had estimated these qualities for the static oscilloscope test but only made a coarse, lower-bound estimate for the others. We knew that the static and phase reversing gratings gave highly correlated results. We suspected that the coarse reliability estimates for the printed tests appeared less reliable than those for the oscilloscope settings only because the latter were based on the mean of 5 scores. We therefore concentrated on the reliability and stability of the 2 printed tests. They represent extreme differences in procedure and factor structure while sharing great practical advantages.

METHOD

We assessed both eyes of 28 normal young adults

(mean age $31 \cdot 1$, SD $6 \cdot 53,12 \mathrm{M}, 16 \mathrm{~F}$ ) on both tests 3 times. The starting eye and test were chosen at random. All testing was completed on the first eye before the other was used. The order of spatial frequencies within each test was randomised, but all 6 were completed as a block before the next test began. The 2 tests were then presented alternately for 3 cycles. Testing procedures were as previously described.

\section{RESULTS}

By treating eyes and spatial frequencies separately we can concentrate on the relations between the 3 repetitions of the 2 tests. From the 6 scores 15 correlations can be calculated. Three are correlations between 4AFC scores on different occasions (the testretest reliability for each 4 AFC plate). Three similarly are for the Arden test. The remaining 9 estimate the between-test correlation. The average of each of these sets at each spatial frequency is shown in Table 6. Clearly the 4AFC test is more reliable than the Arden test, except at the 2 extreme spatial frequencies. Moreover, in the same range its reliability is as good as or better than that determined for oscilloscope settings in the first study (Table 1). The 4AFC test appears less reliable in those conditions where the discs are small or the number of cycles presented very low. Thus it can be attributed to inadequate stimulation and the image degradation from print imperfections and mounting technique. While the problem could be removed in principle, we have seen that these spatial frequencies are the least useful ones.

In the previous experiment a single printed test measure appeared less consistent than the average of 5 oscilloscope settings. In this study, when the 3 replications were averaged first and the same parameter calculated, the value was 0.747 for the 4AFC test and 0.612 for the Arden test. This 4AFC estimate, based on 3 replications, is better than the highest oscilloscope estimate, based on $5(0.684$ for the moving gratings). Clearly not only is averaging

Table 6 Average of 3 test-retest reliabilities from 3 replications of each plate in the $4 A F C$ and Arden tests and average between-test correlations (based on all 9 pairs). The original correlations are based on the scores from 56 normal young adults' eyes, tested 3 times with each of the printed tests.

Spatial frequency

(cycles per degree)

Average 4AFC reliability $(\mathrm{n}=3)$

Average Arden reliability $(\mathbf{n}=3)$

Average intercorrelation

$(\mathrm{n}=9)$ $\begin{array}{llllll}0.1 & 0.2 & 0.4 & 0.8 & 1.6 & 3.2\end{array}$

$\begin{array}{llllll}0.69 & 0.75 & 0.74 & 0.82 & 0.69 & 0.65\end{array}$

$\begin{array}{llllll}0.76 & 0.51 & 0.48 & 0.59 & 0.66 & 0.74\end{array}$

$\begin{array}{llllll}0.27 & 0.36 & 0.22 & 0.12 & 0.09 & 0.34\end{array}$ 
several replications the key to increased reliability, but by both estimation procedures the 4AFC test's reliability equals or exceeds that of the other 3 .

While reliability is the tendency for a subject to retain the same score relative to the others in the group when the test is repeated, stability implies that the mean and standard deviation of the group does not change when the test is repeated. In the Arden test there is a significant tendency for subjects to appear more sensitive on successive trials, but it is small $(0.75$ units of the arbitrary scale, or $1.2 \mathrm{db})$ and becomes significant only when the results of all spatial frequencies are combined. For this reason it was previously reported to be insignificant. ${ }^{16}$ The trend is insignificant for the 4AFC test under either analysis.

Analysis of other aspects of the data, not explicitly related to the results of successive testing, served simply to confirm the results of the previous experiment. Mean scores and variances were similar. Correlations between tests were of the same magnitude and had the same factor structure whether raw scores or averages were considered. Because the different factor structures are consistent, the tests must measure somewhat different faculties.

\section{Experiment 4}

Having established that printed tests gave reliable, stable measures and that the 4AFC format and procedure indeed appeared to be an improvement, we wanted to see if it was also better in practice. Clinically useful tests should have generalisable normal values, as defined above, that is, the normal average score and range of variability should not change with age (or socioeconomic group or motivation level). Moreover it should be efficient, that is, people judged clinically to be mildly affected or at risk should frequently fall outside these limits.

We assessed both eyes of many normal subjects (using both tests once only), until we had sufficiently large numbers in 3 age ranges. We included in this series subjects used in the 2 previous experiments. Only the first set of measures from experiment 3 was used. There were 21 children $(9 \mathrm{M}, 12 \mathrm{~F})$ between 3 and 15 years of age (mean age 8.73, SD 3.64), 55 adults $(25 \mathrm{M}, 30 \mathrm{~F}$ ) between 22 and 49 (mean age $32 \cdot 9$, SD 8.7) and 21 elderly people $(11 \mathrm{M}, 10 \mathrm{~F})$ between 50 and 82 (mean age 60.9, SD 8.75). We also assessed both eyes of 24 glaucomatous patients $(13 \mathrm{M}, 11 \mathrm{~F})$ between 52 and 83 years of age (mean age 65.25, SD
Fig. 3 Age changes in the Arden test (open circles) and the $4 A F C$ test (black circles) at each spatial frequency. Stars indicate significant age trends. Vertical bars represent one standard deviation. Note that there is a consistent and mostly significant trend for elderly people to perform more poorly on the Arden test, while this occurs only at the highest spatial frequency for the $4 A F C$ test. Also note that standard deviations increase with spatial frequency.

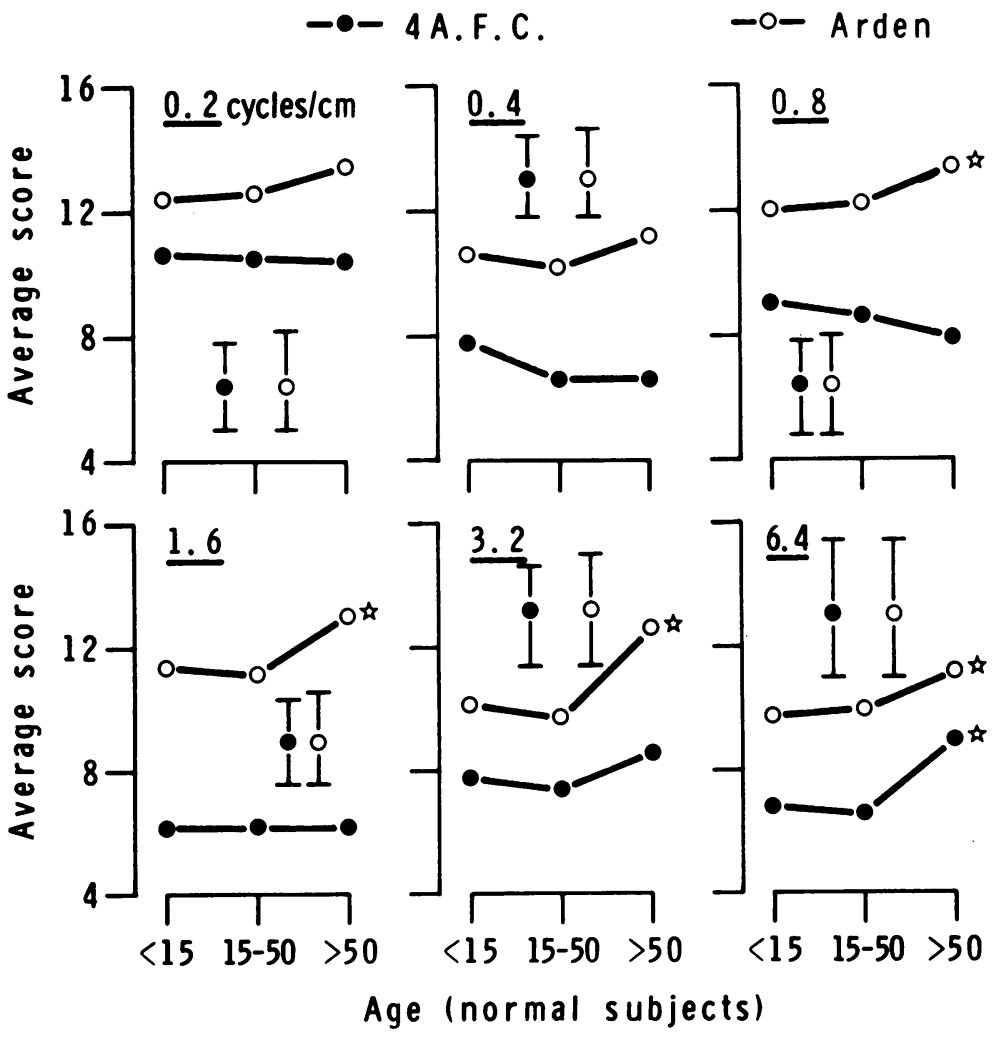


10.35). In all cases at least one eye was clinically judged to be only mildly affected.

The group of patients included 8 symptom-free ocular hypertensives and 3 cases of chronic simple glaucoma, with no loss of visual function or clinical signs except raised pressures and slight cupping in either eye. Of these 11,4 were receiving medication, which was a miotic in 3 instances. The other patients were diagnosed as chronic simple glaucoma (7), closed-angle glaucoma (5), and Posner-Schlossman syndrome (1). The pressure was controlled surgically (3 cases), by miotic drugs (3), nonmiotic drugs (3), both surgery and miotic drugs (1), and surgery and nonmiotic drugs (2). In 3 cases only 1 eye was being treated. Visual symptoms of these 13 ranged from no (4) to at worst moderate visual loss in the better eye and moderate to severe loss in the worst eye. Moderate here means a field loss intruding no closer than 10 degrees to the macula, associated with cupping of the discs, raised intraocular pressures, and a drop in far visual acuity to no less than $0 \cdot 5$. Seven eyes were severe, that is, with field loss including the macula. These 7 eyes failed to detect any plate on either test, and the scores therefore simply reduced the significance of any differences which were observed.

\section{RESULTS}

We first looked for any age trend in the scores of all normal subjects (Fig. 3). Sensitivity only decreased with age at the highest spatial frequency in the 4AFC test. However, there was a clear decrease of the Arden test scores with age at all spatial frequencies. Analysis of variance showed this effect to be insignificant for plates 2 and 3 . Variance again increased with spatial frequency.

We then compared the mean scores of the glaucoma patients with the age matched, normal, elderly control group for each plate separately (Fig. 4, top) and established the significance of these differences by analysis of variance. The glaucoma patients had significantly reduced sensitivity to each
(21)
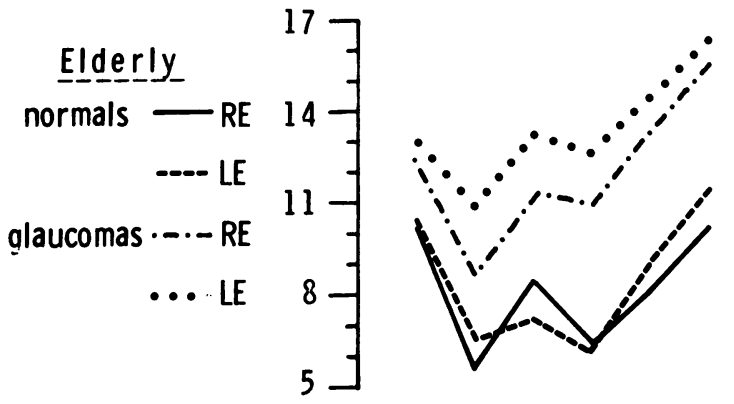

Elderly

(21)

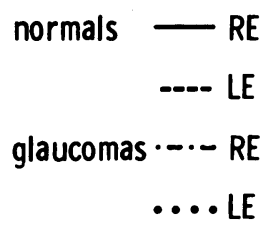

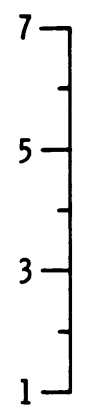
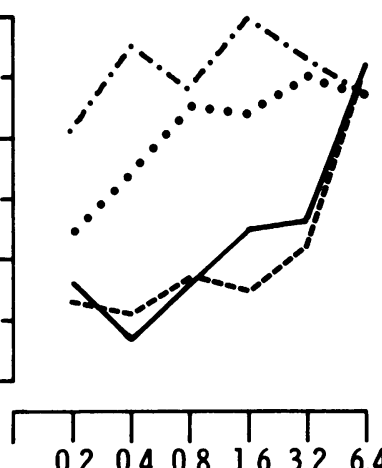
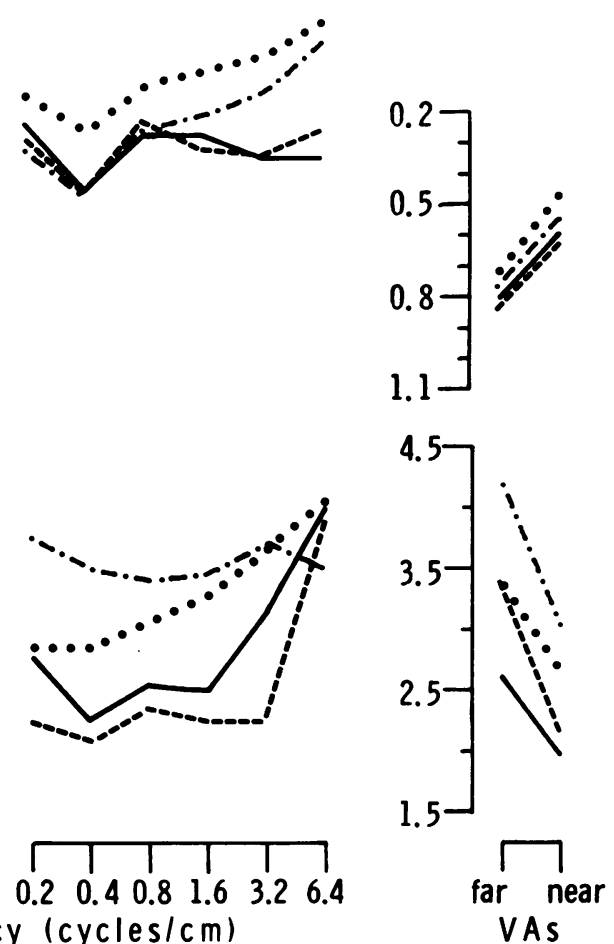

Fig. 4 Means (top row) and standard deviations (bottom row) for right and left eyes of the normal and glaucomatous elderly patients tested on the 4AFC test (left column) and the Arden test (middle column). Near and far visual acuities are given at the far right. Note that the difference between patients and normal persons can be seen even in the visual acuity scores and is slightly greater at high spatial frequencies in both printed tests, but the effect is very small. In the 4AFC test there is a general loss averaging about 4 Arden units $(6 \cdot 4 \mathrm{db})$ across all spatial frequencies. The standard deviations are high partly because 7 eyes with macular involvement have been included, and this has reduced the difference between the 2 tests. 
Fig. 5 A nonparametric measure of signal detection sensitivity (2 arcsine (square root $P(A))$ ) plotted as a measure of information efficiency for various subsets of the measures taken on elderly normal or glaucomatous patients. The measure relates separations of all subjects based on the data subset to the original clinical categories. If a large number of assumptions are made, this score is monotonically but nonlinearly related to the better known criterion of percentage correct, the scale for which is given on the right. Note that visual acuity detects patients more poorly than either contrast sensitivity test, and that one plate of the $4 A F C$ test is more effective than the complete

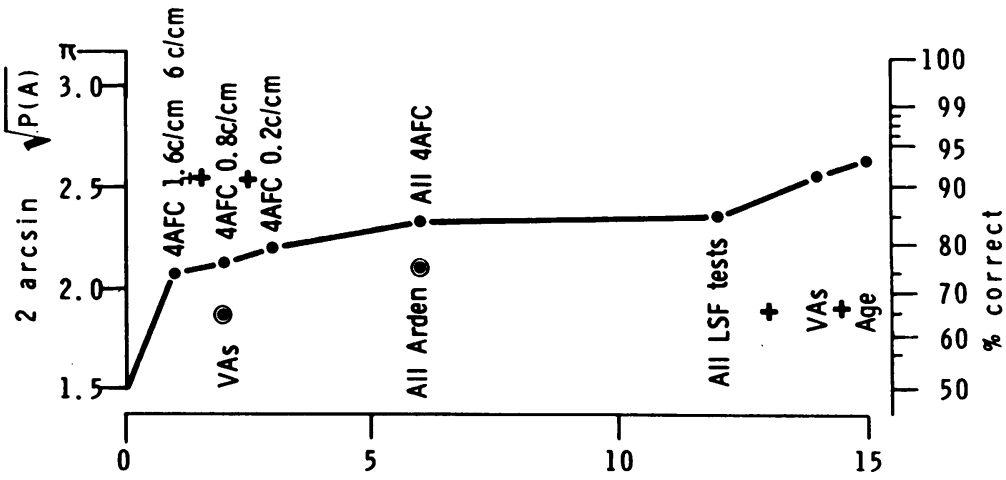
Arden test.

separate $4 \mathrm{AFC}$ plate averaging about 4 Arden units $(6.4 \mathrm{db})$, which is not unlike the difference found in experiment 1 , with very different equipment and psychophysics. When the Arden test was used, the glaucoma patients appeared to have reduced contrast sensitivity for only the highest spatial frequency plate. There was a significant effect in the Arden test if it was scored as prescribed. ${ }^{12}$ The mean aggregate score for normal elderly people $(50 \cdot 3)$ was significantly below the mean aggregate score for the glaucoma patients $(76 \cdot 2)(p<0 \cdot 001$, DF 88$)$ despite the use of 21 rather than 25 as the default score when plates were not seen.

This analysis of the effects of glaucoma on average score is complicated by the standard deviation for the glaucoma patients' scores being much higher than the normal controls', except at the highest spatial frequency, where it is high for both groups (Fig. 4, bottom). Glaucoma did not increase the standard deviation in experiment 1 . Some of this effect must be due to the inclusion of the 8 eyes with macular involvement in this part of the analysis. They could not detect any grating in either test and were given scores of 21 . The differences between the 2 tests would have been more extreme if these data had not be included.

As in experiment 1 and previous reports ${ }^{14}$ we found with both tests that the mean difference between groups was slightly greater at higher spatial frequencies. The differences between the visual acuities of the normal and glaucomatous subjects, while of the same magnitude as that found in experiment 1 , was not significant here because the sample size was smaller. The finest stimulus used in the contrast sensitivity tests, it should be recalled, was
$3 \cdot 2$ cycles/deg, well below the acuity limit. This demonstrates how a real difference may exist at all spatial frequencies but still appear to be restricted to low ones where variances are less.

Although the mean difference between glaucomatous patients and age matched controls is greater with the 4AFC test, we wanted to show directly how much more diagnostic information it contained*. We first estimated precisely how much more efficient this information is in the formal sense defined above. In Fig. 5 we have plotted both efficiency and predicted percentage correct against increasing numbers of plates for both tests. It is clear that either printed test detects glaucoma more efficiently than a visual acuity test can. Moreover one plate of the 4AFC test on its own is more efficient than the complete Arden test.

\footnotetext{
${ }^{*}$ In this formal analysis we first used stepwise multiple regression to see which variables were most heavily correlated with having glaucoma. Discriminatory analysis was then used. It acts on the set of supplied data (here the selected set of scores) and classifies all the elderly subjects into 2 groups, the size of which was specified to be the same as the original clinical classes. It chooses 2 groups which are as different as possible on the basis of the data available to it. ${ }^{30} \mathrm{We}$ then took the variables in various sets, starting with the most effective in each of the 2 tests, or with visual acuity, and adding more variables in the order given by the stepwise multiple regression.

The discriminatory analysis of any data subset provided us with a mathematically derived classification which could be compared with the original clinical categories in a $2 \times 2$ table. The 4 cells of this table correspond to correct assessments. false alarms, misses, and correct rejections of a signal detection task. ${ }^{33}$ Such tables can be converted into a nonparametric measure of sensitivity. Sensitivity in this sense is a precise measure of the efficiency with which the available information can be used to make a decision. If a large number of assumptions are made, it can also be used to predict the percentage of correct decisions which will be made.
} 
Clinical decisions cannot rely on the calculation of complex formulae. While it must be stressed that this clinical study was a very preliminary trial to see if either test format produced larger differences in mean scores between patients and normal persons, the data can be looked at in more detail. As in experiment 1 we can use the raw scores in association with an arbitrary cut-off to establish approximate correct detection and false positive rates. The Arden test is scored in an entirely comparable manner.

Detection rates were calculated for all 41 eyes without macular involvement and for the 24 affected persons, the eye with the maximum score being used except for the 7 cases with macular involvement. False positive rates were calculated for the 42 elderly normal eyes and (because there is no substantial age trend) for the total normal sample (194 eyes). The figures for the least affected eyes are comparable to those found in study 1 provided we remember that there are different groups involved.

With a cut-off between 11 and 13 most $4 \mathrm{AFC}$ plates alone detected $30-45 \%$ of affected eyes and $45-58 \%$ of affected persons with false positive rates of around $5-7 \%$ for elderly normal persons and $2 \cdot 5-4 \%$ for the total population. If we sum the scores of the middle 4 plates of the 4AFC test, which are the most reliable ones, only 1 of the 42 normal elderly eyes we tested scored 42 or more $(2 \%)$. In the total sample of 198 normal eyes only $11(5 \cdot 6 \%)$ had scores above 44 . If we take an aggregate of 44 as a cut-off, $39 \%$ of the affected eyes and $54 \%$ of the glaucoma patients are detected. Other studies will be required before we can decide whether this level of detection is sufficiently high or can be improved upon, but it is close to the mathematical optimum possible with the sophisticated procedure used to derive Fig. 5. The correct assessment rate is as good as or better than that obtained in experiment 1 with a test which takes almost 5 times as long on more expensive equipment, and the false positive rate is substantially lower. It remains lower even when a much wider age range is included in the normal sample.

We have examined the association between contrast sensitivity scored in this way and the clinical characteristics of 43 of the 48 eyes which were examined. Of the eyes with clear visual losses (apart from the 7 with macular involvement) 10/15 were abnormal. Even when there was no other visual loss $4 / 21$ were abnormal. These 4 included 2 cases with severe loss in the other eye ( 1 closed angle, 1 chronic simple case); one other showed mild cupping, and the last had a highest recorded pressure of $38 \mathrm{mmHg}$. Contrast sensitivity loss can therefore be detected even in the very early stages of glaucoma. In general there was an association with both maximum recorded pressure and presence of any field defect, but perhaps because of the small numbers neither was significant $(p=0 \cdot 15)$. There was no clear association with either the use of miotic medication or a history of surgery, but again the sample is too small to be certain. Certainly significant losses could occur in eyes without either treatment. One eye in which there was substantial field loss but macular sparing failed to detect any 4AFC plate, while all the Arden test scores were apparently within normal limits. Such a case clearly underlines the advantages of forced choice testing methods.

\section{DISCUSSION}

Age related changes in contrast sensitivity are restricted to the $3 \cdot 2$ cycles/deg plate with the 4 AFC procedure. Several previous authors have found that sensitivity decreased with age in the Arden test, ${ }^{12-14} 1^{16}$ particularly with the higher frequency plates. We have confirmed that effect and shown that it generalises to ordinary oscilloscope settings. The sole difference between these studies is that the age trend extends to much lower spatial frequencies if subjective methods are used.

Contrary to these observations Sekuler et al. ${ }^{17} 18$ reported that elderly people lose sensitivity to low but not high spatial frequencies. These authors appear to have carefully validated their data, ${ }^{19}$ but several features of the experiment may have produced a wrong conclusion. Of these the most important is that their elderly group may not be a representative sample. The small number of subjects were carefully selected to have normal visual acuity and may therefore have come from a group with extremely high acuity prior to any age deterioration, without any necessary associated superiority at lower spatial frequencies. Other possible problems with these studies include small sample size, a very rapid rate of contrast change during measurement, the use of descending trials, and a failure to check that the estimated threshold was asymptotic.

W. Barnard, D. Powell, and R. Carter (personal communication), working in our Moorfields Clinic, have established norms for a computer-driven contrast sensitivity test on a large sample covering a wide range of ages. They found that the correlation between age and contrast threshold appeared increasingly above 1.6 cycles/deg, which supports the 4AFC test result. Their test was very similar to that used by Sekuler et al. ${ }^{17} 18$ except that it did not have the biases we find in that study. Contrast was linearly ramped. Beasley et al., ${ }^{24}$ also using a similar testing method on a large sample, found that high spatial frequency deficits increase continuously at ages above 20 . Visual acuity changes with age are well known and regularly found (reviewed by Devaney and Johnson ${ }^{28}$ ). There is a clear neurophysiological 
basis for this commonly observed visual acuity loss. ${ }^{28}$ However, specific losses in the low spatial frequency range, while visual acuity remains within normal limits, have never been established in any clinical group with forced choice methodology and large samples. It seems unlikely that age alone would produce such a pattern.

\section{Conclusions}

There appears to be no special loss of low spatial frequency contrast sensitivity in glaucoma. The overall $6 \mathrm{db}$ loss (a factor of 2 ) found in experiments 1 and 4 with very different tests also adequately accounts for the small differences in visual acuity which did occur. The visual acuity differences are not clinically significant in the individual at risk and reach statistical significance only in large samples. As shown above, the variability of contrast threshold determinations decreases at lower spatial frequencies. In this range factors like poor refraction and normal pupillary and lenticular changes also have less effect on the result. This is the main reason why low spatial frequency contrast sensitivity testing is useful. It is unwise to include stimuli of spatial frequency above 2 cycles/deg in such a test. There is no reason to believe that peripheral retina is more likely to be used.

It is possible that the sample was too small or unrepresentative in many studies which do not find significant differences. ${ }^{49141718}$ Atkin et al. ${ }^{910}$ may similarly have had greater success with phasealternating stimuli because responses to them are more reliable (experiment 2 ).

Our experiments estimate 5 formal criteria which can be defined and measured so that the clinical value of tests can be compared: reliability, stability, generality, efficiency, and utility. The psychophysical method influences responses and ranking in a group more than differences in the stimulus, so that stable responses need not accurately reflect contrast sensitivity, and tests with checks on accuracy must prove clinically superior to those where a subject's report is taken at face value.

The 4AFC test we developed is only a prototype, but it is at least equal to all other testing methods on all the criteria by which a comparison has been made, and it is better than each other method on at least one criterion and the known weaknesses can be easily rectified. At most spatial frequencies a 4AFC measure is less variable and more reliable than one from an adjustment method. It gives scores which, relative to the Arden test, are more consistent across retests, norms which generalise to all age groups, and results which are more efficient in the practical aspects of glaucoma detection. Because it is similar to a sight test chart it has a familiar form, and high scores can be trusted. It is portable, inexpensive, fast, and simple to administer and score. This $4 \mathrm{AFC}$ test will help us design another, better, clinical test.

During this study Vaegan received support from the Garrick Foundation Trust for Research into Child Blindness. the East Anglia Regional Health Authority, and the Golden Nugget Fellowship at the Institute for Ophthalmology. London. He currently holds an MRC fellowship. Equipment support was provided by MRC grant C59 to C. Blakemore and P. G. Watson.

Professor G. B. Arden contributed bv discussion and encouragement to every stage of this study and deserves special thanks. The authors also wish to thank Mr P. G. Watson and Mr J. Cairns for permitting them to examine patients in the clinic at New Addenbrooke's Hospital. Cambridge. and all staff members of that clinic who assisted with the ophthalmic examinations. Caroline Hawthorn-Thwaite and Jennifer French assisted with testing in experiments 2 and 3 respectively. Mr R. Hitchings kindly commented on an earlier version of the manuscript.

\section{References}

1 Arden GB. The importance of measuring contrast sensitivity in cases of visual disturbance. Br J Ophthalmol 1978: 62: 198-20\%.

2 Arden GB. Visual loss in patients with normal visual acuity. Trans Ophthalmol Soc UK 1978: 98: 219-23.

3 Arden GB. Jacobson JJ. A simple grating test of contrast sensitivity. Preliminary results indicate value in screening for glaucoma. Invest Ophthalmol Visual Sci 1978: 17: 23-5.

4 Atkin A. Wolkstein M. Bodis-Wollner I. Anders M. Kels B. Podos S. Interocular comparison of contrast sensitivities in glaucoma patients and suspects. Br J Ophthalmol 1980): 64: 858-62.

5 Cooper RL. Constable IJ. Terrell A. Mass screening for glaucoma and other eve disease using the Arden grating test. Aust J Ophthalmol 1980): 8: 131-7.

6 Editorial: Contrast sensitivity revisited. Br J Ophthalmol 1981: 65: 513-4.

7 Arden. GB. Gucukoglu AG. Grating test of contrast sensitivity in patients with retrobulbar neuritis. Arch Ophthalmol 1978: 96: 1926-9.

8 Skalka HW. Comparison of Snellen acuity. VER acuity. and Arden grating scores in macular and optic nerve diseases. $\mathrm{Br} \mathrm{J}$ Ophthalmol 1980: 64: 24-9.

9 Atkin A. Bodis-Wollner I. Wolkstein M. Moss A. Podos S. Spatiotemporal contrast sensitivities in glaucoma. Invest Ophthalmol Visual Sci 1978: 17 (suppl): 24 (ARVO abstr).

10 Atkin A. Bodis-Wollner I. Wolkstein M. Moss A. Podos S. Abnormalities of central contrast sensitivity in glaucoma. Am J Ophthalmol 1979: 88: 205-11.

11 Singh H. Cooper RL. Alder VA. Crawford GJ. Terrell A. Constable IJ. The Arden grating acuity: effect of age and optical factors in the normal patient, with prediction of the false negative rate in screening for glaucoma. Br J Ophthalmol 1981: 65: 518-24.

12 Hsu-Winges C. Stamper RJ. Sopher M. Arden contrast sensitivity testing in normals, glaucoma suspects and chronic glaucoma patients. Invest Ophthalmol Visual Sci 1980): 19 (suppl): 84 (ARVO abstr).

13. Shalka HW. Effect of age on Arden grating acuity. $\mathrm{Br} J$ Ophthalmol 198(); 64: 21-3.

14 Sokol S. Domar A. Moskowitz A. Utility of the Arden grating test in glaucoma screening: high false positive rate in normals over 50 years of age. Invest Ophthalmol Visual Sci 1980): 19: 1529-3.3.

15 Campbell FW. Green DG. Optical and retinal factors affecting visual resolution. J Physiol 1965: 181: 576-93.

16 Vaegan. The clinical value of printed contrast sensitivity tests. J Phusiol 1980; 300: 76P 
17 Sekuler R. Hutman LP. Owsley CJ. Human aging and spatial vision. Science 1980; 209: 1255-6.

18 Sekuler R. Hutman LP. Spatial vision and aging. I: Contrast sensitivity. J Gerontol 1980; 35: 692-9.

19 Hutman LP. Sekuler R. Spatial vision and aging. II: Criterion effects. J. Gerontol 1980: 35: 700-6.

20 Banks RV. Campbell FW. Hess R. Watson PG. A new treatment for amblyopia. Br Orthoptic J 1978; 35: 1-12.

21 Atkinson J. French J. Braddick $O$. Contrast sensitivity function of preschool children. Br J Ophthalmol 1981: 65: 525-9.

22 Schade $\mathrm{OH}$. Optical and photoelectric analog of the eve. J Opt Soc Am 1956: 46: 721-39.

23 Taylor P. A microprocessor controlled gratings generator. J Phvsiol 1978: 284: 20-21P.

24 Beazley LD. Illingworth DJ. Jahn A. Greer DV. Contrast sensitivity in children and adults. Br J Ophthalmol 1980): 64: 863-6.
25 Ross J. Johnstone JJ. Phase and detection of compound gratings. Vision Res 1980); 20: 189-92.

26 Gstadler RJ. Green DG. Laser interferometric acuity in amblyopia. J Pediatr Ophthalmol 1971: 8: 251-6.

27 Hess RF. Campbell FW. Greenhaigh T. On the nature of the neural abnormality in human amblyopia: neural aberrations and neural sensitivity loss. Pfluegers Arch 1978: 377: 201-7.

28 Devaney KO. Johnson HA. Neuron loss in the aging visual cortex of man. J Gerontol 198(); 35: 836-41.

29 Guilford JP. Psvchometric Methods. New York: McGraw Hill. 1954.

30 Morrison DF. Multivariate Statistical Methods. 2nd ed. New York: McGraw Hill. 1972.

31 Robson JG. Spatial and temporal contrast-sensitivity functions of the visual svstem. J Opt Soc Am 1966; 56: 1141-2.

32 Enroth-Cugell C. Robson JG. The contrast sensitivity of retinal ganglion cells of the cat. J Phvsiol 1966: 187: 517-52

$33 \mathrm{McNicol}$ D. A Primer Of Signal Detection Theorv. London: Allen and Unwin. 1972. 